\title{
Risk factors for poor prognosis in nosocomial in- fective endocarditis
}

\author{
Ji-won Hwang ${ }^{1}$, Seung Woo Park ${ }^{1}$, Eun Jeong Cho² , Ga Yeon Lee ${ }^{1}$, Eun Kyoung Kim ${ }^{1}$ Sung-A Chang ${ }^{1}$, \\ Sung-Ji Park ${ }^{1}$, Sang-Chol Lee ${ }^{1}$, Cheol-In Kang ${ }^{3}$, Doo Ryeon Chung ${ }^{3}$, Kyong Ran Peck ${ }^{3}$, and Jae-Hoon Song ${ }^{3}$
}

${ }^{1}$ Division of Cardiology, Department of Medicine, Heart Vascular and Stroke Institute, Samsung Medical Center, Sungkyunkwan University School of Medicine, Seoul; ${ }^{2}$ Department of Cardiology, National Cancer Center, Goyang; ${ }^{3}$ Division of Infectious Disease, Department of Medicine, Samsung Medical Center, Sungkyunkwan University School of Medicine, Seoul, Korea

Received: March 28, 2016

Revised : August 18, 2016

Accepted: August 23, 2016

Correspondence to

Seung Woo Park, M.D.

Division of Cardiology, Department of Medicine, Heart Vascular and Stroke Institute, Samsung Medical Center, Sungkyunkwan University School of Medicine, 81 Irwon-ro, Gangnam-gu, Seoul 06351, Korea

Tel: $+82-2-3410-3419$

Fax: +82-2-3410-3849

E-mail: parksmc@gmail.com
Background/Aims: The aim of our study was to compare the characteristics of nosocomial infective endocarditis (NIE) with community-acquired infective endocarditis (CIE) and to determine independent risk factors for in-hospital death. Methods: We retrospectively reviewed the medical records of 560 patients diagnosed with infective endocarditis. NIE was defined by a diagnosis made $>72$ hours after hospital admission or within 2 months of hospital discharge.

Results: Among the 560 cases reviewed, 121 were classified as NIE. Compared with patients with CIE, patients with NIE were older (mean \pm SD, $51.30 \pm 18.01$ vs. $59.76 \pm 14.87, p<0.001)$. The in-hospital death rate of the NIE group was much higher than that of the CIE group $(27.3 \%$ vs. $5.9 \%, p<0.001)$. More patients with NIE had central intravenous catheters, and were undergoing hemodialysis $(p<$ 0.001). Methicillin-resistant Staphylococcus aureus (MRSA) was the most common causal microorganism of NIE, and MRSA $(p<0.001)$ and fungus $(p=0.002)$ were more common in NIE compared with CIE. On multiple analysis, age, liver cirrhosis, cancer chemotherapy, central intravenous catheter, hemodialysis, and genitourinary tract manipulation were independent clinical risk factors for NIE. Among the patients with NIE, 33 died during their hospital admission. The independent risk factors for in-hospital death were older age (adjusted odds ratio [OR], 1.04; $95 \%$ confidence interval [CI], 1.01 to 1.07; $p=0.037$ ) and chemotherapy for malignancy (adjusted OR, 3.89; 95\% CI, 1.18 to $12.87 ; p=0.026$ ).

Conclusions: Because of the considerable incidence of NIE and its poor prognosis, we should pay attention to early diagnosis and active management of NIE, especially for older patients and patients receiving chemotherapy.

Keywords: Nosocomial infective endocarditis; Clinical risk factors; Risk factors for in-hospital death

\section{INTRODUCTION}

Over the past 20 years, the etiology, epidemiology, and outcomes of infective endocarditis (IE) have changed [13]. In particular, the population at risk for IE is no longer young people with known rheumatic valvulopathy, but rather now includes elderly people with degener- ative valve disease [4]. In addition, invasive therapeutic interventions such as placement of central intravenous catheters, intracardiac devices, and hemodialysis have increased the risk of nosocomial infections including bacteremia and endocarditis in this population [5-8]. Nosocomial infective endocarditis (NIE) is a serious complication of nosocomial bacteremia and results in 
prolonged hospitalization and high mortality rates [5,7]. NIE has various clinical manifestations that depend on the patient's underlying disease, the causal microorganism, and the presence of complications [9]. As a result, appropriate diagnosis and management of patients with NIE, as well as estimation of prognosis, are very important.

To our knowledge, however, few reports have evaluated the clinical characteristics of NIE in Asia. Therefore, the aim of our study was to describe the clinical characteristics of and risk factors for NIE compared with community-acquired infective endocarditis (CIE) and to determine the associated risk factors for short-term clinical outcomes such as in-hospital mortality.

\section{METHODS}

\section{Patient population and data collection}

All consecutive adult patients ( $\geq 15$ years of age) who were diagnosed with IE between January 2000 and July 2014 at Samsung Medical Center (Seoul, Korea), a tertiary university hospital, were screened for inclusion in this study. We retrospectively reviewed medical records from each of these patients including blood culture results and echocardiographic data. The diagnosis of IE was confirmed by modified Duke criteria (2000) and medical records [10]. A total of 560 patients met the modified Duke criteria for definite or possible IE. From their electronic medical records, additional information was collected including demographic characteristics, underlying medical disease, recent history of predisposing manipulations, microbiological and echocardiographic features, short-term outcomes, surgical treatment, and complications. This study was approved by Institutional Review Board of Samsung Medical Center, and the requirement of informed consent was waived.

\section{Echocardiographic findings}

All patients analyzed in this study underwent transthoracic echocardiography (TTE). Transesophageal echocardiography (TEE) was also performed in most of the patients. We investigated the vegetation site, vegetation size, presence of valve disease, and complications such as abscess or tissue perforation. The vegetation size was measured in various planes according to maximal length and width. The severity of valvular regurgitation was estimated by Doppler examination with semiquantitative standard criteria, and the presence of a perivalvular abscess was identified as an abnormal localized echogenic mass without internal blood flow.

\section{Microbiological etiology}

The microbiological information was obtained from blood cultures, intraoperative heart tissue sample cultures and serological studies. Blood samples for culture were drawn under sterile conditions according to the hospital's standard guideline. The microorganism isolated from blood cultures was considered to be the cause of IE in all patients with clinical manifestations of IE. Culture-negative IE was diagnosed when the patients with clinical manifestations of IE showed negative blood and/or intraoperative cultures.

\section{Surgery}

All information was collected by the attending physicians who established the indication for cardiac surgery according to guideline [3].

\section{Definitions}

IE was classified as NIE if the diagnosis was made $>72$ hours after hospital admission and there was no evidence of infection at the time of admission. Additionally, NIE was diagnosed if IE occurred within 60 days after hospital discharge when a high risk procedure for bacteremia was performed or when any predisposing factor for IE was present during hospitalization [11-15]. The underlying cardiac condition was classified according to the presence of a native valve, congenital heart disease, or a prosthetic valve.

Large vegetations were defined as those equal to or larger than $2 \mathrm{~cm}$ in maximal length [11]. Systemic and pulmonary embolic events as well as mycotic aneurysmal events confirmed on imaging studies such as computed tomography, magnetic resonance imaging, or echocardiography were recorded. The short-term outcome studied was in-hospital death, which was defined as death from any cause during hospitalization [11].

\section{Statistical analysis}

Quantitative variables are reported as mean \pm standard deviation (SD). On univariate analysis, Student $t$ tests 


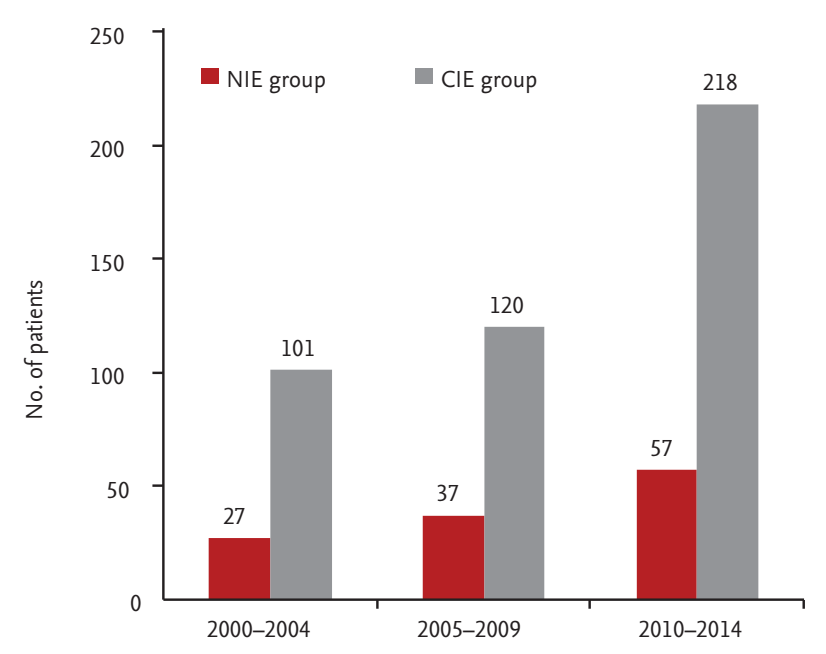

Figure 1. Number of patients with nosocomial infective endocarditis (NIE) and community-acquired infective endocarditis (CIE) from 2000 to 2014 in 5-year intervals.

were used for continuous variables and chi-square tests were used for categorical variables in comparing the distribution of NIE with CIE. $p$ values less than 0.05 were considered to be statistically significant. Clinical risk factors associated with NIE were determined on multiple analysis. Clinical variables affecting in-hospital death that were determined on univariate analysis $(p$ $<0.05$ ) were analyzed by logistic regression in patients with NIE. All analyses were performed using SPSS version 20.0 (IBM Co., Armonk, NY, USA).

\section{RESULTS}

A total of 560 cases of IE were diagnosed between 2000 and 2014. Among them, 121 cases were diagnosed as NIE and 439 as CIE. In assessing cases in 5-year periods over 15 years, we observed an increasing trend in the absolute number of patients with NIE; the 1st, 2nd, and 3rd period contained 27, 37, and 57 cases, respectively (Fig. 1). Likewise, there was a pattern of increase in the number of patients with CIE; the 1st, 2nd, and 3rd period contained 101, 120, and 218 cases, respectively (Fig. 1).

\section{Patients and clinical characteristics}

The clinical characteristics of NIE compared with CIE are summarized in Table 1. NIE patients were older than the CIE patients (mean $\pm \mathrm{SD}, 59.76 \pm 14.87$ years vs.
$51.30 \pm 18.01$ years, $p<0.001)$. With respect to underlying medical diseases, the incidence of liver cirrhosis $(\mathrm{LC})(p$ $=0.002)$, diabetes mellitus $(\mathrm{DM})(p=0.045)$, malignancy (solid and hematologic) $(p<0.001)$, chemotherapy for malignancy $(p<0.001)$, and chronic kidney disease (CKD) $(p<0.001)$ were higher in NIE patients. IE occurring on native valves was more common in the NIE group $(84.3 \%$ vs. $74.9 \%, p=0.038$ ), while IE occurring in patients with congenital heart disease was more frequent in the CIE group ( $4.1 \%$ vs. $21.6 \%, p<0.001$ ). The presence of a central intravenous catheter (33.1\% vs. $2.1 \%, p<0.001$ ) and hemodialysis ( $15.7 \%$ vs. $3.4 \%, p<0.001)$ were more common in the NIE group. Predisposing procedures related with the occurrence of IE were present in 15 of 121 NIE cases (12.4\%), and in 52 of 439 CIE cases (11.8\%) (Table 1). A history of acupuncture or articular injection $(p=0.031)$ and dental manipulation $(p=0.002)$ were more common in the CIE group (Table 1), whereas more specific procedures were more common in NIE patients; four patients in the NIE group had a history of endoscopic esophageal variceal ligation, compared with one patient in the CIE group $(p=0.009)$. Genitourinary tract manipulation was performed in four patients with NIE and two patients with $\operatorname{CIE}(p=0.022)$.

On multiple analysis, age, LC, chemotherapy, presence of a central intravenous catheter, hemodialysis, and genitourinary tract manipulation as predisposing procedure were independently associated with NIE as clinical risk factors (Table 2).

\section{Echocardiographic findings}

Echocardiographic findings for both groups are also shown in Table 1 . The mitral valve was the most common vegetation site in both groups, and its incidence was higher in the CIE group $(p=0.023)$. Vegetation involvement of the right atrium was more common in the NIE group $(p=0.006)$. Both TTE and TEE were performed in 417 patients. The vegetations were found in the 387 patients. Among them, 103 patients were found to have vegetations in TEE only. Among 103 patients, 75 patients $(72.8 \%)$ did not have the significant valvular regurgitant lesion, 88 patients $(85.4 \%)$ had single site involvement of vegetation, and 83 patients $(80.6 \%)$ had small size of vegetation such as $<1 \mathrm{~cm}$. In addition, 38 patients (36.9\%) were belonged to prosthetic valve or repaired valve endocarditis. 
Table 1. Baseline clinical characteristics and clinical outcomes of NIE compared with CIE

\begin{tabular}{|c|c|c|c|}
\hline Characteristic & $\operatorname{CIE}(\mathrm{n}=439)$ & $\operatorname{NIE}(\mathrm{n}=121)$ & $p$ value \\
\hline Age, yr & $51.30 \pm 18.01$ & $59.76 \pm 14.87$ & $<0.001$ \\
\hline Male sex & $281(64.0)$ & $64(52.9)$ & 0.027 \\
\hline Definite infective endocarditis & $312(71.1)$ & $94(77.7)$ & 0.170 \\
\hline \multicolumn{4}{|l|}{ Underlying medical disease } \\
\hline Liver cirrhosis & $14(3.2)$ & $13(10.7)$ & 0.002 \\
\hline Alcoholism & $8(1.8)$ & $4(3 \cdot 3)$ & 0.300 \\
\hline Diabetes mellitus & $32(7 \cdot 3)$ & $16(13.2)$ & 0.045 \\
\hline Chemotherapy & $16(3.6)$ & $35(28.9)$ & $<0.001$ \\
\hline Malignancy & $32(7 \cdot 3)$ & $52(43.0)$ & $<0.001$ \\
\hline Chronic kidney disease & $22(5.0)$ & $20(16.5)$ & $<0.001$ \\
\hline Hemodialysis & $15(3.4)$ & $19(15.7)$ & $<0.001$ \\
\hline Solid organ transplantation & $1(0.2)$ & $1(0.8)$ & 0.390 \\
\hline Coronary artery disease & $13(3.0)$ & $7(5.8)$ & 0.160 \\
\hline Rheumatologic disease & $4(0.9)$ & $2(1.7)$ & 0.620 \\
\hline Cerebrovascular accident & $13(3.0)$ & $2(1.7)$ & 0.750 \\
\hline Cardiomyopathy & $5(1.1)$ & $1(0.8)$ & 1.000 \\
\hline Recurrent infection (biliary, urinary) & $4(0.9)$ & $1(0.8)$ & 1.000 \\
\hline Other site abscess & $11(2.5)$ & $4(3 \cdot 3)$ & 0.540 \\
\hline COPD \& ILD & $5(1.1)$ & $2(1.7)$ & 0.650 \\
\hline Tuberculosis & $15(3.4)$ & $3(2.5)$ & 0.780 \\
\hline Vascular disease (vasculitis) & $3(0.7)$ & $1(0.8)$ & 1.000 \\
\hline \multicolumn{4}{|l|}{ Underlying cardiac condition } \\
\hline Congenital heart disease & $95(21.6)$ & $5(4 \cdot 1)$ & $<0.001$ \\
\hline Prosthetic valve & $84(19.1)$ & $17(14.0)$ & 0.230 \\
\hline Native valve & $329(74.9)$ & $102(84 \cdot 3)$ & 0.038 \\
\hline Central intravenous catheter & $9(2.1)$ & $40(33 \cdot 1)$ & $<0.001$ \\
\hline Past history of infective endocarditis & $31(7.1)$ & $3(3 \cdot 3)$ & 0.200 \\
\hline \multicolumn{4}{|l|}{ Predisposing manipulation } \\
\hline Acupuncture or articular injection & $17(3.9)$ & o & 0.031 \\
\hline Dental manipulation & $26(5.9)$ & o & 0.002 \\
\hline Endoscopy with procedure & $1(0.2)$ & $4(3 \cdot 3)$ & 0.009 \\
\hline TACE or liver biopsy & $2(0.5)$ & $2(1.7)$ & 0.210 \\
\hline Genitourinary tract manipulation & $2(0.5)$ & $4(3 \cdot 3)$ & 0.022 \\
\hline Orthopedic prosthesis & $1(0.2)$ & $2(1.7)$ & 0.120 \\
\hline Perivertebral area procedure & $2(0.5)$ & $2(1.7)$ & 0.210 \\
\hline Intracardiac device (PPM, ICD) & $1(0.2)$ & $1(0.8)$ & 0.390 \\
\hline \multicolumn{4}{|l|}{ Echocardiographic finding } \\
\hline Transesophageal echocardiogram & $342(77 \cdot 9)$ & $75(62.0)$ & $<0.001$ \\
\hline \multicolumn{4}{|l|}{ Vegetation } \\
\hline Mitral valve & $258(58.8)$ & $57(47.1)$ & 0.023 \\
\hline
\end{tabular}


Table 1. Continued

\begin{tabular}{|c|c|c|c|}
\hline Characteristic & $\operatorname{CIE}(\mathrm{n}=439)$ & $\operatorname{NIE}(n=121)$ & $p$ value \\
\hline Aortic valve & $163(37.1)$ & $48(39 \cdot 7)$ & 0.670 \\
\hline Tricuspid valve & $30(6.8)$ & $12(9.9)$ & 0.250 \\
\hline Pulmonary valve & $17(3 \cdot 9)$ & $1(0.8)$ & 0.140 \\
\hline Right ventricle & $15(3.4)$ & $1(0.8)$ & 0.210 \\
\hline Right atrium & $7(1.6)$ & $8(6.6)$ & 0.006 \\
\hline Other site & $22(5 \cdot 0)$ & $6(5.0)$ & 1.000 \\
\hline Significant valvular regurgitation & $214(48.7)$ & $40(33.1)$ & 0.003 \\
\hline Large vegetation $(>2 \mathrm{~cm})$ & $34(7 \cdot 7)$ & $10(8.3)$ & 0.750 \\
\hline Perivalvular abscess & $60(13.7)$ & $15(12.4)$ & 0.770 \\
\hline Perforation & $36(8.2)$ & $7(5.8)$ & 0.450 \\
\hline \multicolumn{4}{|l|}{ Causative organism } \\
\hline Viridans group streptococci & $128(29.2)$ & $8(6.6)$ & $<0.001$ \\
\hline \multicolumn{4}{|l|}{ Staphylococcus aureus } \\
\hline Methicillin-sensitive & $63(14.4)$ & $19(15 \cdot 7)$ & 0.770 \\
\hline Methicillin-resistant & $22(5 \cdot 0)$ & $28(23.1)$ & $<0.001$ \\
\hline Enterococcus & $31(7 \cdot 1)$ & $15(12.4)$ & 0.060 \\
\hline Coagulase-negative Staphylococcus & $19(4 \cdot 3)$ & $11(9.1)$ & 0.060 \\
\hline HACEK & $7(1.6)$ & 0 & 0.360 \\
\hline Fungus & $2(0.5)$ & $6(5.0)$ & 0.002 \\
\hline Other bacteria & $55(12.5)$ & $20(16.5)$ & 0.290 \\
\hline Culture negative infective endocarditis & $129(29.4)$ & $16(13.2)$ & $<0.001$ \\
\hline \multicolumn{4}{|l|}{ Clinical outcomes } \\
\hline In-hospital death & $26(5 \cdot 9)$ & $33(27 \cdot 3)$ & $<0.001$ \\
\hline Cardiac surgery & $298(67.9)$ & $46(38.0)$ & $<0.001$ \\
\hline On indication of cardiac surgery (but not done) & $41(9 \cdot 3)$ & $29(24.0)$ & $<0.001$ \\
\hline Embolic event & $157(35.8)$ & $48(39.7)$ & 0.460 \\
\hline Brain & $118(26.9)$ & $34(28.1)$ & 0.820 \\
\hline Kidney & $14(3.2)$ & $5(4 \cdot 1)$ & 0.580 \\
\hline Spleen & $21(4.8)$ & $6(5 \cdot 0)$ & 1.000 \\
\hline Lung & $18(4.1)$ & $7(5.8)$ & 0.460 \\
\hline Extremities & $14(3.2)$ & $6(5.0)$ & 0.410 \\
\hline Liver & $2(0.5)$ & $1(0.8)$ & 0.520 \\
\hline Eye (retina) & $6(1.4)$ & $2(1.7)$ & 0.690 \\
\hline Mycotic aneurysm & $20(4 \cdot 5)$ & $5(4 \cdot 1)$ & 1.000 \\
\hline Duration of hospitalization, day & $35.80 \pm 21.70$ & $47.33 \pm 37.59$ & $<0.001$ \\
\hline
\end{tabular}

Values are presented as mean \pm SD or number (\%).

NIE, nosocomial infective endocarditis; CIE, community-acquired infective endocarditis; COPD, chronic obstructive pulmonary disease; ILD, interstitial lung disease; TACE, transcatheter arterial chemoembolization; PPM, permanent pacemaker; ICD, implantable cardioverter defibrillator; HACEK, Haemophilus parainfluenzae, Haemophilus aphrophilus, Aggregatibacter actinomycetemcomitans, Cardiobacterium hominis, Eikenella corrodens, and Kingella kingae. 
Table 2. Univariate and multiple analysis of clinical factors associated with nosocomial infective endocarditis

\begin{tabular}{|c|c|c|c|c|}
\hline \multirow{2}{*}{ Variable } & \multicolumn{2}{|c|}{ Crude } & \multicolumn{2}{|c|}{ Adjusted } \\
\hline & OR $(95 \% \mathrm{CI})$ & $p$ value & OR $(95 \% \mathrm{CI})^{\mathrm{a}}$ & $p$ value \\
\hline Age & $1.03(1.02-1.04)$ & $<0.001$ & $1.02(1.001-1.04)$ & 0.006 \\
\hline Male sex & $1.58(1.06-2.38)$ & 0.027 & $1.43(0.86-2.38)$ & 0.170 \\
\hline Liver cirrhosis & $3.65(1.67-8.00)$ & 0.001 & $4.57(1.86-11.21)$ & 0.001 \\
\hline Diabetes mellitus & $1.94(1.03-3.67)$ & 0.042 & $1.47(0.67-3.25)$ & 0.340 \\
\hline Chemotherapy & $10.76(5.70-20.31)$ & $<0.001$ & $5.03(2.29-11.06)$ & $<0.001$ \\
\hline Central intravenous catheter & $23.59(11.0-50.50)$ & $<0.001$ & $10.18(4.21-24.62)$ & $<0.001$ \\
\hline Hemodialysis & $5.27(2.59-10.72)$ & $<0.001$ & $2.58(1.02-6.53)$ & 0.046 \\
\hline Endoscopy with procedure & $14.97(1.66-135.25)$ & 0.016 & $10.53(0.98-13.54)$ & 0.052 \\
\hline Genitourinary tract manipulation & $7.47(1.35-41.59)$ & 0.021 & $11.22(1.84-68.36)$ & 0.009 \\
\hline
\end{tabular}

OR, odds ratio; CI, confidence interval.

${ }^{a}$ Estimated by multiple linear regression analysis of the variables indicated in the table.

Table 3. Clinical characteristics in dead patients compared with survived patient for the group of NIE and CIE by univariate analysis

\begin{tabular}{|c|c|c|c|c|c|c|}
\hline \multirow[b]{2}{*}{ Characteristic } & \multicolumn{3}{|c|}{ NIE } & \multicolumn{3}{|c|}{ CIE } \\
\hline & $\begin{array}{c}\text { Survived } \\
(\mathrm{n}=88)\end{array}$ & $\begin{array}{c}\text { Died } \\
(\mathrm{n}=33)\end{array}$ & $p$ value & $\begin{array}{c}\text { Survived } \\
(\mathrm{n}=413)\end{array}$ & $\begin{array}{c}\text { Died } \\
(n=26)\end{array}$ & $p$ value \\
\hline Age, yr & $58.08 \pm 15.72$ & $64.24 \pm 11.36$ & 0.020 & $50.34 \pm 17.62$ & $66.54 \pm 17.65$ & $<0.001$ \\
\hline Male sex & $52(59.1)$ & $12(36.4)$ & 0.040 & $264(63.9)$ & $17(65 \cdot 4)$ & 1.000 \\
\hline \multicolumn{7}{|l|}{ Underlying medical disease } \\
\hline Liver cirrhosis & $9(10.2)$ & $4(12.1)$ & 0.750 & $12(2.9)$ & $2(7 \cdot 7)$ & 0.200 \\
\hline Alcoholism & $4(4 \cdot 5)$ & o & 0.570 & $8(1.9)$ & o & 1.000 \\
\hline Diabetes mellitus & $14(15 \cdot 9)$ & $2(6.1)$ & 0.230 & $31(7 \cdot 5)$ & $1(3.8)$ & 0.710 \\
\hline Chemotherapy & $20(22.7)$ & $15(45 \cdot 5)$ & 0.023 & $15(3.6)$ & $1(3.8)$ & 1.000 \\
\hline Malignancy & $32(36.4)$ & $20(60.6)$ & 0.023 & $28(6.8)$ & $4(15 \cdot 4)$ & 0.110 \\
\hline Chronic kidney disease & $18(20.5)$ & $2(6.1)$ & 0.100 & $19(4 \cdot 6)$ & $3(11.5)$ & 0.130 \\
\hline Hemodialysis & $17(19 \cdot 3)$ & $2(6.1)$ & 0.100 & $13(3.1)$ & $2(7 \cdot 7)$ & 0.220 \\
\hline Solid organ transplantation & 0 & $1(3.0)$ & 0.270 & $1(0.2)$ & o & 1.000 \\
\hline Coronary artery disease & $6(6.8)$ & $1(3.0)$ & 0.670 & $12(2.9)$ & $1(3.8)$ & 0.550 \\
\hline Rheumatologic disease & $1(1.1)$ & $1(3.0)$ & 1.000 & $4(1.0)$ & o & 1.000 \\
\hline Cerebrovascular accident & $1(1.1)$ & $1(3.0)$ & 1.000 & $13(3.1)$ & 0 & 1.000 \\
\hline Cardiomyopathy & $1(1.1)$ & o & 1.000 & $4(1.0)$ & $1(3.8)$ & 0.260 \\
\hline Recurrent infection (biliary, urinary) & $1(1.1)$ & 0 & 1.000 & $3(0.7)$ & $1(3.8)$ & 0.220 \\
\hline Other site abscess & $4(4 \cdot 5)$ & o & 0.570 & $9(2.2)$ & $2(7 \cdot 7)$ & 0.130 \\
\hline COPD \& ILD & 0 & $2(6.1)$ & 0.070 & $5(1.2)$ & 0 & 1.000 \\
\hline Tuberculosis & $3(3 \cdot 4)$ & o & 0.560 & $15(3.6)$ & o & 1.000 \\
\hline Vascular disease (vasculitis) & $1(1.1)$ & 0 & 1.000 & $3(0.7)$ & o & 1.000 \\
\hline \multicolumn{7}{|l|}{ Underlying cardiac condition } \\
\hline Congenital heart disease & $4(4 \cdot 5)$ & $1(3.0)$ & 1.000 & $95(23.0)$ & o & 0.002 \\
\hline
\end{tabular}


Table 3. Continued

\begin{tabular}{|c|c|c|c|c|c|c|}
\hline \multirow[b]{2}{*}{ Characteristic } & \multicolumn{3}{|c|}{ NIE } & \multicolumn{3}{|c|}{ CIE } \\
\hline & $\begin{array}{c}\text { Survived } \\
(\mathrm{n}=88)\end{array}$ & $\begin{array}{c}\text { Died } \\
(\mathrm{n}=33)\end{array}$ & $p$ value & $\begin{array}{c}\text { Survived } \\
(\mathrm{n}=413)\end{array}$ & $\begin{array}{c}\text { Died } \\
(n=26)\end{array}$ & $p$ value \\
\hline Prosthetic valve & $12(13.6)$ & $5(15.2)$ & 0.780 & $76(18.4)$ & $8(30.8)$ & 0.130 \\
\hline Native valve & $15(17.0)$ & $4(12.1)$ & 0.590 & $308(74.6)$ & $21(80.8)$ & 0.640 \\
\hline On indication of cardiac surgery (but not done) & $16(18.2)$ & $13(39 \cdot 4)$ & 0.030 & $24(5.8)$ & $17(65 \cdot 4)$ & $<0.001$ \\
\hline Central intravenous catheter & $28(31.8)$ & $12(36.4)$ & 0.670 & $8(1.9)$ & $1(3.8)$ & 0.430 \\
\hline Past history of infective endocarditis & $1(1.1)$ & $3(9.1)$ & 0.060 & $29(7 \cdot 0)$ & $2(7 \cdot 7)$ & 0.700 \\
\hline \multicolumn{7}{|l|}{ Predisposing manipulation } \\
\hline Acupuncture or articular injection & o & o & NA & $17(4 \cdot 1)$ & o & 0.610 \\
\hline Dental manipulation & 0 & o & NA & $24(5.8)$ & $2(7 \cdot 7)$ & 0.660 \\
\hline Endoscopy with procedure & $4(4 \cdot 5)$ & ० & 0.570 & $1(0.2)$ & ० & 1.000 \\
\hline TACE or liver biopsy & $1(1.1)$ & $1(3.0)$ & 0.470 & $2(0.5)$ & o & 1.000 \\
\hline Genitourinary manipulation & $3(3.4)$ & $1(3.0)$ & 1.000 & $2(0.5)$ & o & 1.000 \\
\hline Orthopedic prosthesis & $2(2.3)$ & 0 & 1.000 & $1(0.2)$ & ० & 1.000 \\
\hline Perivertebral area procedure & $2(2.3)$ & o & 1.000 & $2(0.5)$ & o & 1.000 \\
\hline Intracardiac device (PPM, ICD) & $1(1.1)$ & ० & 1.000 & $1(0.2)$ & o & 1.000 \\
\hline \multicolumn{7}{|l|}{ Causative organism } \\
\hline Viridans group streptococci & $5(5 \cdot 7)$ & $3(9.1)$ & 0.680 & $127(30.8)$ & $1(3.8)$ & 0.002 \\
\hline \multicolumn{7}{|l|}{ Staphylococcus aureus } \\
\hline Methicillin-sensitive & $19(21.6)$ & 0 & 0.002 & $59(14.3)$ & $4(15 \cdot 4)$ & 0.780 \\
\hline Methicillin-resistant & $17(19 \cdot 4)$ & $11(33 \cdot 3)$ & 0.150 & $17(4 \cdot 1)$ & $5(19.2)$ & 0.006 \\
\hline Enterococcus & $8(9.1)$ & $7(21.2)$ & 0.120 & $28(6.8)$ & $3(11.5)$ & 0.420 \\
\hline Coagulase-negative Staphylococcus & $10(11.4)$ & $1(3.0)$ & 0.290 & $18(4 \cdot 4)$ & $1(3.8)$ & 1.000 \\
\hline HACEK & O & o & NA & $7(1.7)$ & o & 1.000 \\
\hline Fungus & $2(2.3)$ & $4(12.1)$ & 0.046 & $1(0.2)$ & $1(3.8)$ & 0.120 \\
\hline Other bacteria & $16(18.2)$ & $4(12.1)$ & 0.590 & $52(12.6)$ & $3(11.5)$ & 1.000 \\
\hline Culture negative & $11(12.8)$ & $4(12.1)$ & 0.410 & $121(29 \cdot 3)$ & $8(30.8)$ & 0.830 \\
\hline
\end{tabular}

Values are presented as mean \pm SD or number (\%).

NIE, nosocomial infective endocarditis; CIE, community-acquired infective endocarditis; COPD, chronic obstructive pulmonary disease; ILD, interstitial lung disease; NA, not assessed; TACE, transcatheter arterial chemoembolization; PPM, permanent pacemaker; ICD, implantable cardioverter defibrillator; HACEK, Haemophilus parainfluenzae, Haemophilus aphrophilus, Aggregatibacter actinomycetemcomitans, Cardiobacterium hominis, Eikenella corrodens, and Kingella kingae.

\section{Causative microorganisms of NIE}

Blood culture data for the NIE and CIE groups are also presented in Table 1. Overall, blood culture positive IE was more frequently observed in the NIE group. Viridans group streptococci were the most frequent microorganisms involved in CIE ( $\mathrm{n}=128$ cases, 29.2\%), followed by Staphylococcus aureus ( $\mathrm{n}=85$ cases, 19.4\%). However, S. aureus was the most frequent pathogen in the NIE group ( $\mathrm{n}=47$ cases, $38.3 \%$ ), with methicillin-resistant Staphylo- coccus aureus (MRSA) as the major pathogen. Fungus was also a common cause of NIE.

\section{Clinical outcomes}

Clinical outcomes such as in-hospital death, frequency of cardiac surgery, and occurrence of an embolic event or mycotic aneurysm are shown in Table 1 . The in-hospital death rate of the NIE group was much higher than that of the CIE group (27.3\% vs. 5.9\%, $p<0.001$ ). Cardi- 
Table 4. Association between clinical characteristics and in-hospital death in patients with nosocomial infective endocarditis by multiple analysis

\begin{tabular}{|c|c|c|c|c|}
\hline \multirow{2}{*}{ Variable } & \multicolumn{2}{|c|}{ Crude } & \multicolumn{2}{|l|}{ Adjusted } \\
\hline & OR $(95 \% \mathrm{CI})$ & $p$ value & OR $(95 \% \mathrm{CI})^{\mathrm{a}}$ & $p$ value \\
\hline Age & $1.03(1.01-1.06)$ & 0.045 & $1.04(1.01-1.07)$ & 0.037 \\
\hline Male sex & $0.40(0.17-0.90)$ & 0.028 & $0.42(0.17-1.08)$ & 0.070 \\
\hline Liver cirrhosis & $1.21(0.35-4.24)$ & 0.770 & $2.80(0.64-12.34)$ & 0.170 \\
\hline Chemotherapy & $2.83(1.21-6.61)$ & 0.016 & $3.89(1.18-12.87)$ & 0.026 \\
\hline Hemodialysis & $0.27(0.06-1.24)$ & 0.090 & $0.36(0.07-1.91)$ & 0.230 \\
\hline Central intravenous catheter & $1.22(0.53-2.83)$ & 0.640 & $0.82(0.26-2.63)$ & 0.740 \\
\hline On indication of cardiac surgery (but not done) & $2.93(1.21-7.08)$ & 0.017 & $2.18(0.81-5.88)$ & 0.120 \\
\hline Fungus & $5.93(1.03-34.09)$ & 0.046 & $4.43(0.61-31.94)$ & 0.140 \\
\hline
\end{tabular}

OR, odds ratio; CI, confidence interval.

${ }^{a}$ Estimated by multiple linear regression analysis of the variables indicated in the table.

ac surgery was conducted for the treatment of IE significantly less often in cases of NIE than in cases of CIE (38.0\% vs. $67.9 \%, p<0.001$ ). Furthermore, the number of cases that had surgical indication for management, but had not undergone surgery, was higher in the NIE group than in the CIE group (24.0\% vs. 9.3\%, $p<0.001$ ). A total of 157 cases (35.8\%) in the CIE group and 48 cases (39.7\%) in the NIE group experienced embolic events during their hospitalization $(p=0.460)$. The duration of hospitalization was longer in NIE group $(47.33 \pm 37.59$ days vs. $35.80 \pm 21.70$ days, $p<0.001$ ).

The association between clinical characteristics and in-hospital death in patients with NIE is depicted in Tables 3 and 4. On univariate analysis (Table 3), the factors associated with in-hospital death in patients with NIE were age, gender, cancer chemotherapy, surgical indication but not performed, and fungal pathogens. However, on multiple logistic regression (Table 4), only older age (adjusted odds ratio [OR], 1.04; 95\% confidence interval [CI], 1.01 to 1.07; $p=0.037$ ) and chemotherapy treatment for malignancy (adjusted OR, 3.89; 95\% CI, 1.18 to 12.87; $p$ $=0.026$ ) remained as independent risk factors associated with in-hospital mortality.

In patients with CIE, the association between clinical characteristics and in-hospital death is depicted in Tables 3 and 5, as the same method of analysis was used for NIE. On univariate analysis (Table 3), the factors associated with in-hospital death in patients with CIE were age, indication of cardiac surgery but not performed, and causative pathogens like as viridans group streptococci and MRSA. However, on multiple logistic regression (Table 5), it was concluded that older age (adjusted OR, 1.04; 95\% CI, 1.01 to 1.07; $p=0.012$ ), indication of cardiac surgery but not done (adjusted OR, 29.83; 95\% CI, 10.19 to 87.31; $p<0.001$ ), and MRSA (adjusted OR, 9.58; $95 \% \mathrm{CI}, 2.27$ to $40.45 ; p=0.002$ ) as virulent pathogen were clinically significant variables in the analysis.

\section{DISCUSSION}

In recent years, there have been major changes in the epidemiology of IE, including increased age of patients, presence of predisposing factors, and higher rates of NIE, which is related to more frequent use of intravascular devices and procedures $[1,5,16]$. NIE reported in other studies accounts for $10 \%$ to $30 \%$ of all IE cases, similar to what was seen in our study ( $21.6 \%$ of all patients) $[2,9,11,17-19]$. This finding could be explained by increases in the number of nosocomial blood stream infections, the number of patients admitted to intensive care units, the number of invasive vascular procedures performed, and in the higher incidence of degenerative valve disease in the aged population [11,15,18-24]. In addition, other reasons for the growing numbers of reported NIE cases include the greater diagnostic yield of TEE [25] and extension of incubation periods to culture a greater range of causal organisms [5]. 
Table 5. Association between clinical characteristics and in-hospital death in patients with community-acquired infective endocarditis by multiple analysis

\begin{tabular}{|c|c|c|c|c|}
\hline \multirow{2}{*}{ Variable } & \multicolumn{2}{|l|}{ Crude } & \multicolumn{2}{|l|}{ Adjusted } \\
\hline & Crude OR (95\% CI) & $p$ value & Adjusted OR $(95 \% \text { CI })^{\mathrm{a}}$ & $p$ value \\
\hline Age & $1.06(1.03-1.09)$ & $<0.001$ & $1.04(1.01-1.07)$ & 0.012 \\
\hline Male sex & $1.07(0.46-2.45)$ & 0.880 & $1.09(0.38-3.16)$ & 0.870 \\
\hline Liver cirrhosis & $2.79(0.59-13.15)$ & 0.200 & $1.13(0.17-7.30)$ & 0.900 \\
\hline On indication of cardiac surgery (but not done) & $30.62(12.36-75.84)$ & $<0.001$ & $29.83(10.19-87.31)$ & $<0.001$ \\
\hline Viridans group streptococci & $0.09(0.01-0.67)$ & 0.019 & $0.14(0.01-1.16)$ & 0.070 \\
\hline MRSA & $5.55(1.87-16.49)$ & 0.002 & $9.58(2.27-40.45)$ & 0.002 \\
\hline
\end{tabular}

OR, odds ratio; CI, confidence interval; MRSA, methicillin-resistant Staphylococcus aureus.

${ }^{\text {a }}$ Estimated by multiple linear regression analysis of the variables indicated in the table.

In this study, we found that the ratio of NIE cases remained stationary over three 5-year periods. However, the absolute number of patients with NIE has increased continuously. The growing problem of NIE as a large part of all IE cases calls for more careful monitoring of complications after procedures and more appropriate management of immunosuppressed patients, especially in light of recent increased frequencies of invasive procedures and or aggressive medical management.

The clinical features of NIE and its prognosis were evaluated in the current study. There was significant association of NIE with central intravenous catheter-related infections, hemodialysis, and underlying disease such as LC, DM, malignancy, chemotherapy for malignancy, and CKD. Among these factors, increased age, LC, malignancy, and presence of a central intravenous catheter were independently associated with NIE on multivariate analysis, suggesting an increased frequency of NIE in immunocompromised patients as above. Also, these underlying diseases could predispose the patient to the development of concomitant infections due to associated immunosuppression, more invasive procedures, and more frequent hospitalizations [9,19].

The procedural source of infection determined the causative organism in cases of NIE. When the source was an intravascular device or procedure, staphylococci were the most frequent etiologic agent $[12,26,27]$. In NIE patients of our study, the microbiological etiology was analyzed by predisposing procedures. With endoscopic procedures, two cases of viridans group streptococci, one case of methicillin-sensitive Staphylococcus aureus, and one case of enterococcus were identified. Two cases of MRSA were found, one in transcatheter arterial chemoembolization, and other in liver biopsy. Two cases as MRSA in the procedure of orthopedic prosthesis, and one case of MRSA with intracardiac device was recognized. It showed staphylococcal infection was dominant in NIE. In the procedure of genitourinary tract manipulation, however, three cases of enterococcus among four patients were found. Furthermore, in our study, the greater frequency of a central intravenous catheter (33.1\% vs. $2.1 \%, p<0.001$ ) and hemodialysis $(15.7 \%$ vs. $3.4 \%, p<0.001)$ in the NIE group could explain why the most common etiologic agent causing NIE was MRSA, which was found in $23.1 \%$ of patients of the NIE group.

Despite advances in medical and surgical treatment, mortality rates for IE have remained unchanged over the last 20 years, which can be explained by the remarkable epidemiologic shift that now includes elderly patients with multiple comorbidities [9]. Our study showed that patients with NIE were frailer than those with CIE, as indicated by their underlying health issues. In-hospital mortality was higher in the NIE group than in the CIE group. Older age was an independent clinical factor in both groups that determined in-hospital mortality. Among other associated risk factors for in-hospital mortality, chemotherapy treatment was the other independent clinical risk factor for the NIE group. In the CIE group, MRSA as the causative pathogen was an additional clinical risk factor that determined in-hospital mortality. These results suggest that the mortality of NIE is influenced mainly by patients' general health 
conditions, whereas the virulence of the disease-causing pathogen is an additional important mortality factor of CIE.

The percentage of patients who were indicated for but did not undergo cardiac surgery was higher in the NIE group than the CIE group (Table 1). In another study [19], cardiac surgery that was indicated but not performed was an associated clinical risk factor in patients with NIE. However, it was not confirmed as an independent risk factor in the current study. This could be because other mortality related risk factors, such as older age and serious underlying disease, were the main causes for patients not to undergo cardiac surgery. This could also be explained by the small number of this subset of patients in the NIE group.

Our study has several limitations. It is subject to referral bias since it was conducted at a large tertiary care medical center and is retrospective in design. Although increase in the number of NIE patients was shown, there was no proportional increase of NIE patients. The authors do not have a correct answer to explain the phenomenon. There may be a proportional increase of invasive procedures in response to increase of high risk patients for IE. However, it needs a multicenter prospective study to find out the answer.

Some of the underlying diseases may be associated with the in-hospital mortality in patients with NIE, but we could not confirm any independent associations of these underlying diseases with mortality. This might be because of the small number of patients with each underlying disease. However, the total 560 cases enrolled in our study between 2000 and 2014 were larger than those of other studies [18,21].

In conclusion, the incidence of NIE is not low, and the general in-hospital mortality of NIE is quite high. Therefore, it is important that clinicians, particularly those in tertiary care hospital centers where a great number of ambulatory procedures (e.g., hemodialysis and chemotherapy for malignancy) are performed, be aware of the risk of endocarditis in patients who have undergone invasive procedures that could potentially cause bacteremia. Prompt diagnosis by echocardiography and active management would be warranted if NIE is suspected.

\section{KEY MESSAGE}

1. It is important that clinicians, particularly those in tertiary care hospital centers where a great number of ambulatory procedures are performed, be aware of the risk of endocarditis in patients who have undergone invasive procedures that could potentially cause bacteremia.

2. Prompt diagnosis by echocardiography and active management would be warranted if nosocomial infective endocarditis is suspected.

\section{Conflict of interest}

No potential conflict of interest relevant to this article was reported.

\section{REFERENCES}

1. Fefer P, Raveh D, Rudensky B, Schlesinger Y, Yinnon AM. Changing epidemiology of infective endocarditis: a retrospective survey of 108 cases, 1990-1999. Eur J Clin Microbiol Infect Dis 2002;21:432-437.

2. Prendergast BD. The changing face of infective endocarditis. Heart 2006;92:879-885.

3. Habib G, Hoen B, Tornos P, et al. Guidelines on the prevention, diagnosis, and treatment of infective endocarditis (new version 2009): the Task Force on the Prevention, Diagnosis, and Treatment of Infective Endocarditis of the European Society of Cardiology (ESC). Endorsed by the European Society of Clinical Microbiology and Infectious Diseases (ESCMID) and the International Society of Chemotherapy (ISC) for Infection and Cancer. Eur Heart J 2009;30:2369-2413.

4. Castillo JC, Anguita MP, Ruiz M, et al. Changing epidemiology of native valve infective endocarditis. Rev Esp Cardiol 2011;64:594-598.

5. Fernandez-Guerrero ML, Verdejo C, Azofra J, de Gorgolas M. Hospital-acquired infectious endocarditis not associated with cardiac surgery: an emerging problem. Clin Infect Dis 1995;20:16-23.

6. Gouello JP, Asfar P, Brenet O, Kouatchet A, Berthelot G, Alquier P. Nosocomial endocarditis in the intensive care unit: an analysis of 22 cases. Crit Care Med 2000;28:377382 . 
7. Gilleece A, Fenelon L. Nosocomial infective endocarditis. J Hosp Infect 2000;46:83-88.

8. Gregoratos G. Infective endocarditis in the elderly: diagnosis and management. Am J Geriatr Cardiol 2003;12:183189.

9. Fernandez-Hidalgo N, Tornos Mas P. Epidemiology of infective endocarditis in Spain in the last 20 years. Rev Esp Cardiol (Engl Ed) 2013;66:728-733.

10. Li JS, Sexton DJ, Mick N, et al. Proposed modifications to the Duke criteria for the diagnosis of infective endocarditis. Clin Infect Dis 2000;30:633-638.

11. Martin-Davila P, Fortun J, Navas E, et al. Nosocomial endocarditis in a tertiary hospital: an increasing trend in native valve cases. Chest 2005;128:772-779.

12. Fernandez-Hidalgo N, Almirante B, Tornos P, et al. Contemporary epidemiology and prognosis of health care-associated infective endocarditis. Clin Infect Dis 2008;47:1287-1297.

13. Durante-Mangoni E, Utili R. Nosocomial endocarditis: still a challenging diagnosis. Intern Emerg Med 2014;9:715-716.

14. Kiefer TL, Bashore TM. Infective endocarditis: a comprehensive overview. Rev Cardiovasc Med 2012;13:e105-e120.

15. Ben-Ami R, Giladi M, Carmeli Y, Orni-Wasserlauf R, Siegman-Igra Y. Hospital-acquired infective endocarditis: should the definition be broadened? Clin Infect Dis 2004;38:843-850.

16. Cabell $\mathrm{CH}$, Jollis JG, Peterson GE, et al. Changing patient characteristics and the effect on mortality in endocarditis. Arch Intern Med 2002;162:90-94.

17. Mouly S, Ruimy R, Launay O, et al. The changing clinical aspects of infective endocarditis: descriptive review of 90 episodes in a French teaching hospital and risk factors for death. J Infect 2002;45:246-256.

18. Giannitsioti E, Skiadas I, Antoniadou A, et al. Nosocomial vs. community-acquired infective endocarditis in Greece: changing epidemiological profile and mortality risk. Clin Microbiol Infect 2007;13:763-769.

19. Lomas JM, Martinez-Marcos FJ, Plata A, et al. Healthcare-associated infective endocarditis: an undesirable effect of healthcare universalization. Clin Microbiol Infect 2010;16:1683-1690.

20. Nienaber JJ, Kusne S, Riaz T, et al. Clinical manifestations and management of left ventricular assist device-associated infections. Clin Infect Dis 2013;57:1438-1448.

21. Nunez Aragon R, Pedro-Botet Montoya ML, Mateu Prunonosa L, et al. Associated factors and descriptive analysis of healthcare-related infective endocarditis in a tertiary reference hospital. Enferm Infecc Microbiol Clin 2013;31:15-22.

22. Ruiz M, Sanchez MP, Dominguez JC, et al. Infective endocarditis in patients receiving chronic hemodialysis: clinical features and outcome. J Heart Valve Dis 2005;14:11-14.

23. Farinas MC, Llinares P, Almirante B, et al. New trends in infective endocarditis. Enferm Infecc Microbiol Clin 2011;29 Suppl 4:22-35.

24. Peetermans WE, Hill EE, Herijgers $P$, et al. Nosocomial infective endocarditis: should the definition be extended to 6 months after discharge. Clin Microbiol Infect 2008;14:970-973.

25. Pujol M, Hornero A, Saballs M, et al. Clinical epidemiology and outcomes of peripheral venous catheter-related bloodstream infections at a university-affiliated hospital. J Hosp Infect 2007;67:22-29.

26. Hsu RB. Risk factors for nosocomial infective endocarditis in patients with methicillin-resistant Staphylococcus aureus bacteremia. Infect Control Hosp Epidemiol 2005;26:654-657.

27. Benito N, Miro JM, de Lazzari E, et al. Health care-associated native valve endocarditis: importance of non-nosocomial acquisition. Ann Intern Med 2009;150:586-594. 